CRYSTALLOGRAPHIC COMMUNICATIONS

ISSN 2056-9890

Received 1 July 2016

Accepted 7 July 2016

Edited by W. T. A. Harrison, University of Aberdeen, Scotland

Keywords: crystal structure; coumarin; carboxamide; Hirshfeld surface analysis.

CCDC reference: 1491340

Supporting information: this article has supporting information at journals.iucr.org/e

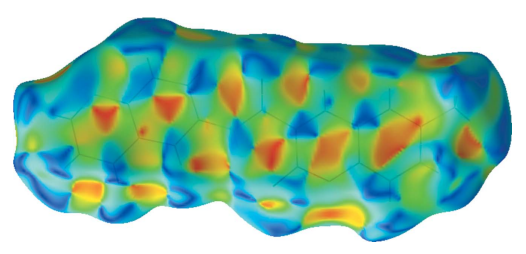

OPEN $\odot$ ACCESS

\section{6-Methyl-2-oxo- $\mathrm{N}$-(quinolin-6-yl)-2H-chromene-3- carboxamide: crystal structure and Hirshfeld surface analysis}

\author{
Lígia R. Gomes, ${ }^{\text {a,b }}$ John Nicolson Low, ${ }^{\mathrm{c} *}$ André Fonseca, ${ }^{\mathrm{d}}$ Maria João Matos ${ }^{\mathrm{d}}$ and \\ Fernanda Borges ${ }^{\text {d }}$
}

a FP-ENAS-Faculdade de Ciências de Saúde, Escola Superior de Saúde da UFP, Universidade Fernando Pessoa, Rua Carlos da Maia, 296, P-4200-150 Porto, Portugal, '⿳⺈冂大 REQUIMTE, Departamento de Química e Bioquímica, Faculdade de Ciências da Universidade do Porto, Rua do Campo Alegre, 687, P-4169-007 Porto, Portugal, 'Department of Chemistry, University of Aberdeen, Meston Walk, Old Aberdeen AB24 3UE, Scotland, and ${ }^{\mathrm{d} C I Q U P / D e p a r t a m e n t o ~ d e ~ Q u i ́ m i c a ~ e ~}$ Bioquímica, Faculdade de Ciências, Universidade do Porto, 4169-007 Porto, Portugal. *Correspondence e-mail: jnlow111@gmail.com

The title coumarin derivative, $\mathrm{C}_{20} \mathrm{H}_{14} \mathrm{~N}_{2} \mathrm{O}_{3}$, displays intramolecular $\mathrm{N}-\mathrm{H} \cdots \mathrm{O}$ and weak $\mathrm{C}-\mathrm{H}$... O hydrogen bonds, which probably contribute to the approximate planarity of the molecule [dihedral angle between the coumarin and quinoline ring systems $\left.=6.08(6)^{\circ}\right]$. The supramolecular structures feature $\mathrm{C}-\mathrm{H} \cdots \mathrm{O}$ hydrogen bonds and $\pi-\pi$ interactions, as confirmed by Hirshfeld surface analyses.

\section{Chemical context}

Coumarin and its derivatives are widely recognized by their unique biological properties (Matos et al., 2014; VazquezRodriguez et al., 2013; Chimenti et al., 2010). Our work in this area has shown that coumarin is a valid scaffold for the development of new drugs for aging related diseases, specifically within the class of monoamino oxidase B inhibitors (Matos et al., 2009). On the other hand, quinoline is a nitrogen heterocycle also often used in drug-discovery programs due to its remarkable biological properties, some of them related to neurodegenerative diseases (Sridharan et al., 2011), for instance, as $\gamma$-secretase and acetylcholinesterase inhibitors (Camps et al., 2009). As part of our ongoing studies in this area (Gomes et al., 2016), we describe the synthesis and crystal structure of the title coumarin-quinoline hybrid, 6-methyl-2oxo- $N$-(quinolin-6-yl)-2H-chromene-3-carboxamide, (1) (see Scheme).<smiles>Cc1ccc2oc(=O)c(C(=O)Nc3ccc4ncccc4c3)cc2c1</smiles>

(1)

\section{Structural commentary}

Fig. 1 shows an ellipsoid plot of the molecular structure of (1). An inspection of the bond lengths shows that there is a slight 
Table 1

Hydrogen-bond geometry $\left(\AA,^{\circ}\right)$.

\begin{tabular}{lllll}
\hline$D-\mathrm{H} \cdots A$ & $D-\mathrm{H}$ & $\mathrm{H} \cdots A$ & $D \cdots A$ & $D-\mathrm{H} \cdots A$ \\
\hline $\mathrm{C} 314-\mathrm{H} 314 \cdots \mathrm{O} 31^{\mathrm{i}}$ & 0.95 & 2.50 & $3.278(2)$ & 139 \\
$\mathrm{C} 8-\mathrm{H} 8 \cdots \mathrm{N} 311^{\mathrm{ii}}$ & 0.95 & 2.68 & $3.394(3)$ & 133 \\
$\mathrm{C} 317-\mathrm{H} 317 \cdots \mathrm{O} 31$ & 0.95 & 2.29 & $2.903(2)$ & 122 \\
$\mathrm{~N} 32-\mathrm{H} 32 \cdots \mathrm{O} 2$ & $0.907(18)$ & $1.879(18)$ & $2.686(2)$ & $147.3(15)$ \\
\hline
\end{tabular}

Symmetry codes: (i) $x+1, y, z$; (ii) $x-\frac{1}{2},-y+\frac{1}{2}, z-\frac{1}{2}$; (iii) $x-1, y, z$.

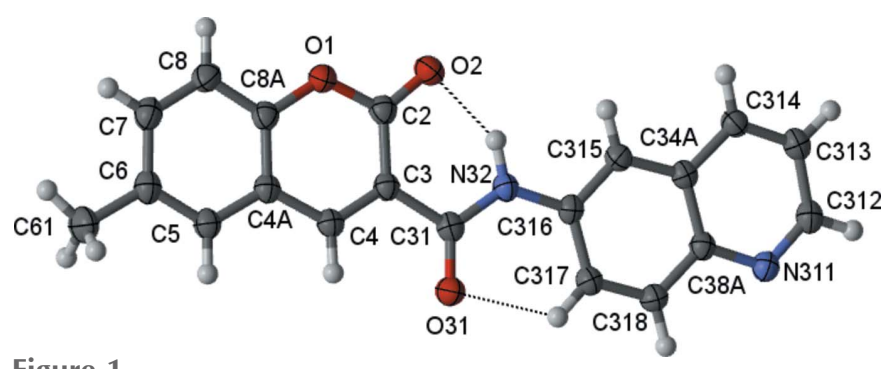

Figure 1

A view of the asymmetric unit of (1), showing the atom-numbering scheme. Displacement ellipsoids are drawn at the $70 \%$ probability level.

asymmetry of the electronic distribution around the coumarin

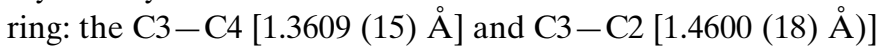
bond lengths are shorter and longer, respectively, than those expected for a $\mathrm{C}_{\mathrm{ar}}-\mathrm{C}_{\mathrm{ar}}$ bond, suggesting that the electronic density is rather located near the $\mathrm{C} 3-\mathrm{C} 4$ bond at the pyrone ring, as occurs in other coumarin-3-carboxamide derivatives (Gomes et al., 2016). Also, the C3-C31 bond length [1.5075 (18) $\AA$ ] is similar to the mean value displayed by other coumarin-3-carboxamide derivatives previously characterized (Gomes et al., 2016) and is of the same order as a Csp ${ }^{3}-\mathrm{Csp}^{3}$ bond.

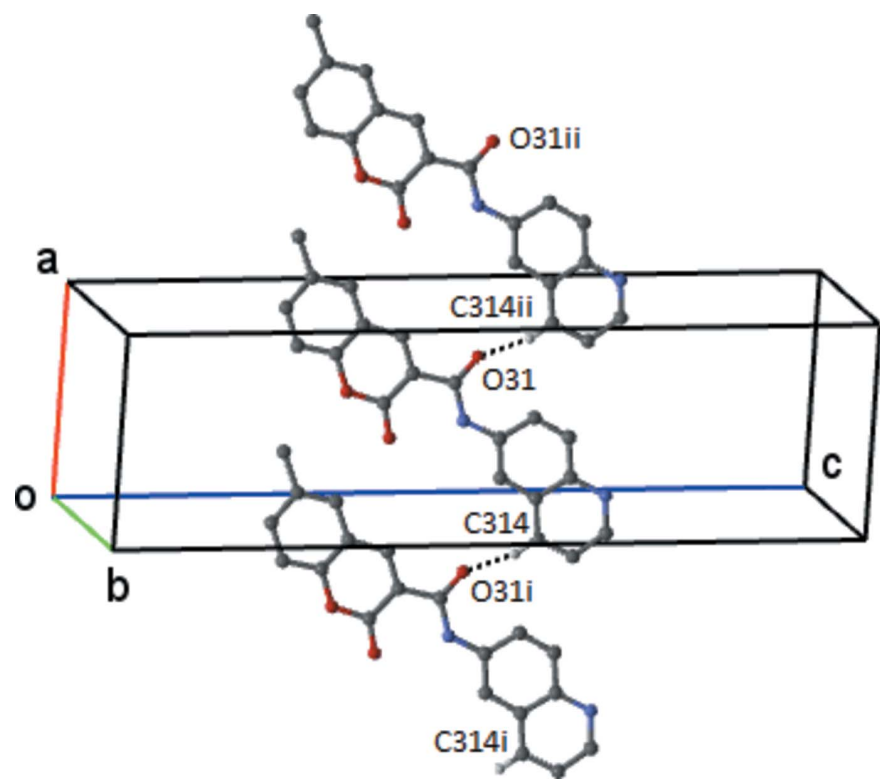

Figure 2

The simple $C 4$ chain in compound (1) formed by the weak C314$\mathrm{H} 314 \cdots \mathrm{O}^{\mathrm{i}}$ hydrogen bond. This chain extends by unit translation along the $a$ axis. $\mathrm{H}$ atoms not involved in the hydrogen bonding have been omitted. [Symmetry codes: (i) $x-1, y, z$; (ii) $x+1, y, z$.]
Table 2

Selected dihedral angles $\left(^{\circ}\right)$.

\begin{tabular}{llll}
\hline Compound & $\theta_{1}\left({ }^{\circ}\right)$ & $\theta_{2}\left({ }^{\circ}\right)$ & $\theta_{3}\left({ }^{\circ}\right)$ \\
\hline$(1)$ & $6.08(6)$ & $5.0(12)$ & $1.73(11)$ \\
\hline
\end{tabular}

Notes: $\theta_{1}$ is the dihedral angle between the mean planes of the coumarin and quinoline rings; $\theta_{2}$ is the dihedral angle between the mean plane of the coumarin ring and the plane defined by atoms $\mathrm{O} 31 / \mathrm{C} 31 / \mathrm{N} 32 ; \theta_{3}$ is the dihedral angle between the mean plane of the quinoline ring and the plane defined by atoms $\mathrm{O} 31 / \mathrm{C} 31 / \mathrm{N} 32$.

The $\mathrm{C}-\mathrm{N}$ rotamer of the amide group governs the conformation of the molecule: the -anti orientation where the $\mathrm{N}$ atom is -cis positioned with respect to the oxo $\mathrm{O}$ atom of the coumarin system allows the establishment of an intramolecular $\mathrm{N} 32-\mathrm{H} 32 \cdots \mathrm{O} 2$ hydrogen bond between the amino group of the carboxamide and the oxo group of the coumarin system, and of a weak intramolecular C317-H317 ..OO31 hydrogen bond that connects the quinoline ring with the $\mathrm{O}$ atom of the carboxamide group (Table 1). Both these interactions form $S(6)$ rings and connect the spacer carboxamide group with the heteroaromatic rings, probably constraining the rotation/bending of those rings with respect to the plane formed by the amide atoms. In fact, the molecule is roughly planar, as may be evaluated by the set of values for the dihedral angles which are less than $7^{\circ}$ (Table 2).

\section{Supramolecular features}

In the crystal of (1), molecules are linked by a weak C314$\mathrm{H} 314 \cdots \mathrm{O} 31^{\mathrm{i}}$ hydrogen bond to form a $C(8)$ chain, which runs parallel to the $a$ axis (Fig. 2 and Table 1). There are several $\pi-\pi$ contacts that will be described below.

\section{Hirshfeld surface analyses}

The Hirshfeld surfaces and two-dimensional fingerprint (FP) plots (Rohl et al., 2008) were generated using Crystal Explorer (Wolff et al., 2012). Compound (1) has three $\mathrm{O}$ atoms and an $\mathrm{N}$ atom that can potentially act as acceptors for hydrogen bonds, but one of the lone pairs of the oxo $\mathrm{O}$ atoms of the coumarin nucleus and of the amide moiety are involved in the establishment of intramolecular hydrogen bonds, as discussed above. As such, they contribute to the electronic density of the pro-molecule in the calculation of the Hirshfeld surface, leaving only the remaining pairs available for participation in the supramolecular structure formation. The surface mapped over $d_{\text {norm }}$ displays several red spots that correspond to areas of close contacts between the surface and the neighbouring environment, and the FP plot is presented in Fig. 3.

The contributions from various contacts, listed in Table 3, were selected by partial analysis of the FP plot. Taking out the $\mathrm{H} \cdot \mathrm{H}$ contacts on the surface that are inherent to organic molecules, the most significant contacts can be divided in three groups: (i) $\mathrm{H} \cdots \mathrm{O} / \mathrm{O} \cdots \mathrm{H}$ together with $\mathrm{H} \cdots \mathrm{N} / \mathrm{N} \cdots \mathrm{H}$ that correspond to weak $\mathrm{C}-\mathrm{H} \cdots \mathrm{O} / \mathrm{N}$ intermolecular interactions $(24.5 \%$ ); (ii) $\mathrm{C} \cdots \mathrm{C}$ and $\mathrm{N} \cdots \mathrm{C} / \mathrm{C} \cdots \mathrm{N}$ contacts that are related 

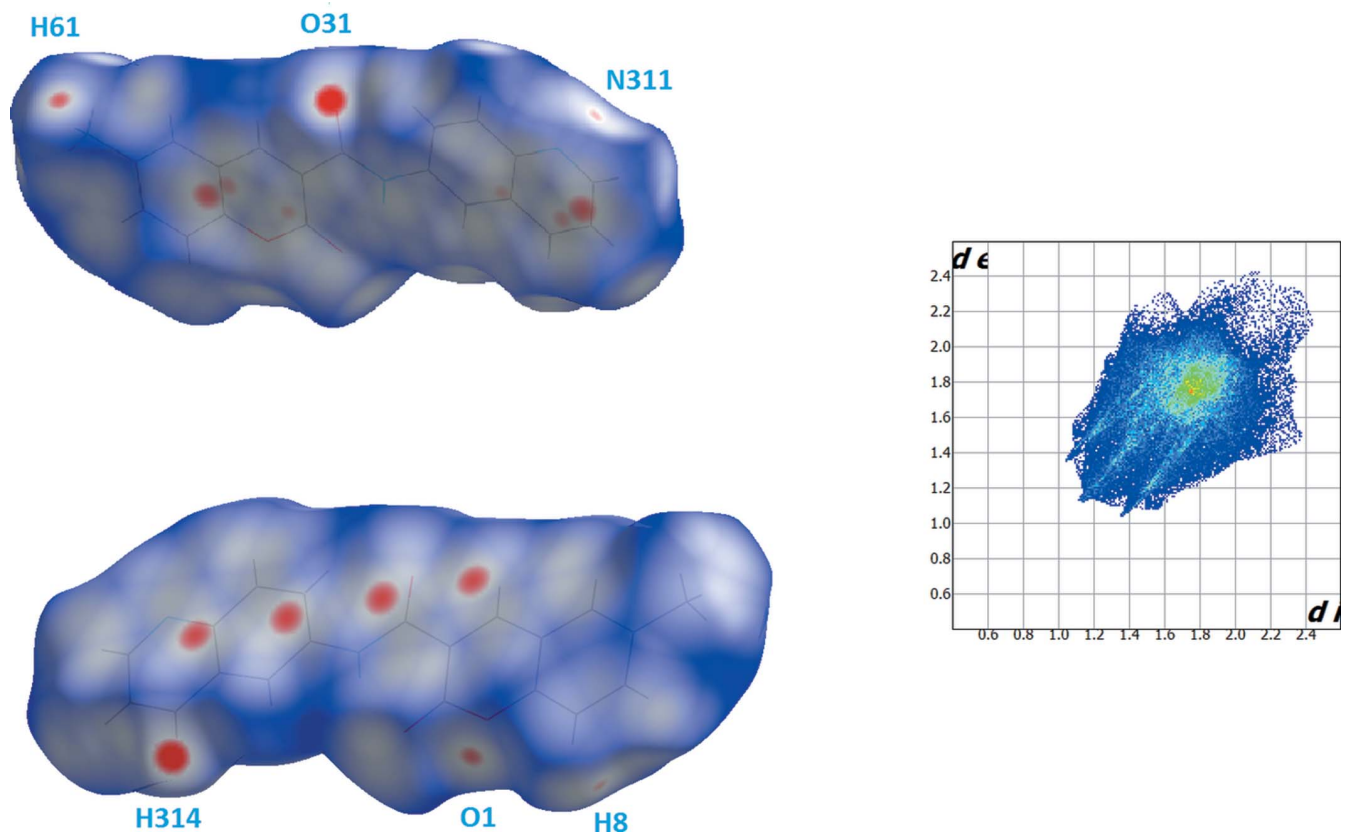

Figure 3

Views of the Hirshfeld surface mapped over $d_{\text {norm }}$ (left) and fingerprint plot (right, FP) for (1). The highlighted red spots on the top face of the surfaces indicate contact points with the atoms participating in the intermolecular $\mathrm{C}-\mathrm{H} \cdots \mathrm{O}$ interactions, whereas those on the middle of the surface corresponds to $\mathrm{C} \cdots \mathrm{C}$ contacts consequent of the $\pi-\pi$ stacking. The $\mathrm{C} \cdots \mathrm{C}$ contacts contribute to the higher frequency of the pixels at $d_{\mathrm{e}} / d_{\mathrm{i}}$ at $1.8^{\circ}$ on the FP plot (yellow spot). The FP plot displays two light-blue spikes (external ends corresponding to $\mathrm{C} \cdots \mathrm{H}$ contacts).

with $\pi-\pi$ stacking (17.9\%): (iii) $\mathrm{H} \cdots \mathrm{C} / \mathrm{C} \cdots \mathrm{H}$ contacts $(14.3 \%)$.

The $\mathrm{H} \cdots \mathrm{N} / \mathrm{O}$ contacts appear as three highlighted red spots on the top and bottom edges of the surface which form pairs of spots of comlementary size, indicating the contact points of the
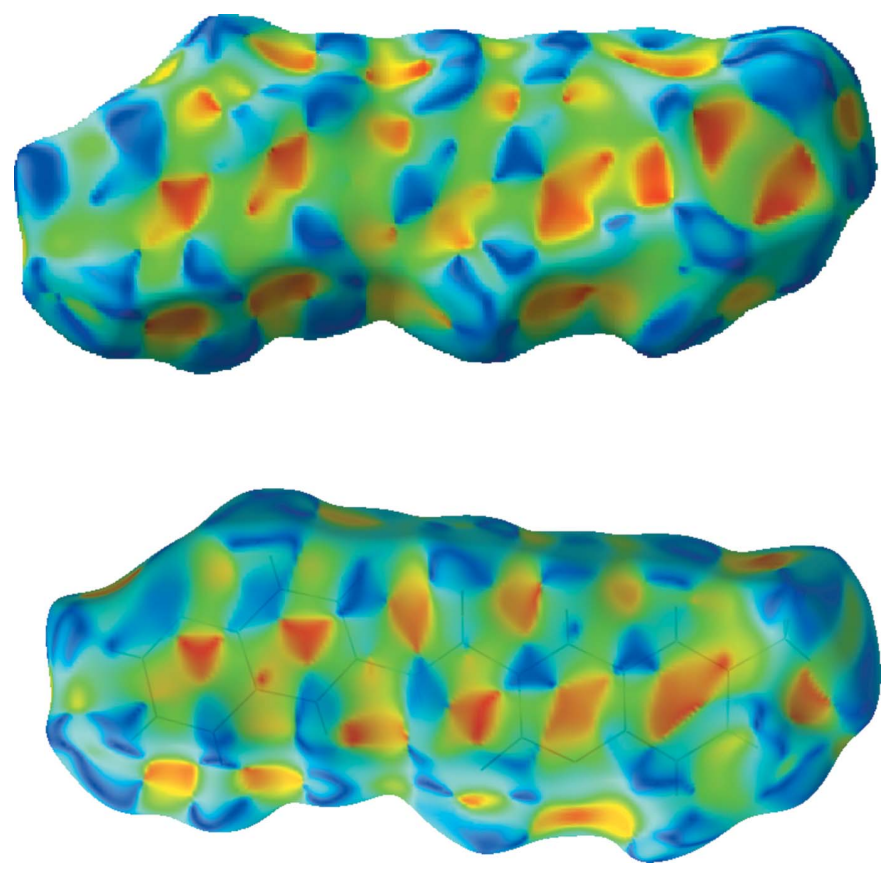

Figure 4

Shape index plots showing the interactions arising from $\pi-\pi$ stacking. The upper corresponds to the stacking across $\left(\frac{1}{2}, 1, \frac{1}{2}\right)$, while the lower corresponds to the stacking across $\left(\frac{1}{2}, \frac{1}{2}, \frac{1}{2}\right)$.
Table 3

Percentages of atom-atom contacts for (1) (\%).

\begin{tabular}{lllllll}
\hline Contact & $\mathrm{H} \cdots \mathrm{H}$ & $\begin{array}{l}\mathrm{H} \cdots \mathrm{O} / \\
\mathrm{O} \cdots \mathrm{H}\end{array}$ & $\begin{array}{l}\mathrm{H} \cdots \mathrm{N} / \\
\mathrm{N} \cdots \mathrm{H}\end{array}$ & $\mathrm{C} \cdots \mathrm{C}$ & $\begin{array}{l}\mathrm{N} \cdots \mathrm{C} / \\
\mathrm{C} \cdots \mathrm{N}\end{array}$ & $\begin{array}{l}\mathrm{H} \cdots \mathrm{C} / \\
\mathrm{C} \cdots \mathrm{H}\end{array}$ \\
\hline$(\%)$ & 40.6 & 21.2 & 3.3 & 13.2 & 4.7 & 14.3 \\
\hline
\end{tabular}

labelled atoms participating in the $\mathrm{C}-\mathrm{H} \cdots \mathrm{N} / \mathrm{O}$ interactions (Fig. 3). The strongest spots correspond to oxo atom O31 of the carboxamide acceptor and donor atom H314, which forms the $\mathrm{C} 314-\mathrm{H} 314 \cdots \mathrm{O} 31^{\mathrm{i}}$ hydrogen bond (Table 1), and the other spots correspond to very weak hydrogen-bond contacts, one involving pyrone atom $\mathrm{O} 1$ and a $\mathrm{H}$ atom of the methyl group (C61-H61B $\cdots \mathrm{O} 1^{\mathrm{ii}}$; Table 1$)$, and the other appearing perpendicular to the quinoline $\mathrm{N}$ atom indicating a very weak $\mathrm{C} 8-\mathrm{H} 8 \cdots \mathrm{N} 311^{\mathrm{ii}}$ contact (Table 1 ). In spite of the weakness of these contacts, their relative strength is reflected in the FP plots where the pair of sharp spikes pointing to south-west is highlighted in light blue.

In this structure, $\mathrm{C} / \mathrm{N} \cdots \mathrm{C}$ contacts prevail over the $\mathrm{C}-$ $\mathrm{H} \cdot \mathrm{C}$ ones. In fact, the packing in (1) is built up by several $\pi-$ $\pi$ interactions (Table 4 ). The red spots in the frontal zone of the surface correspond to these close contacts. Furthermore, the FP plot also reveals an intense cluster at $d_{\mathrm{e}} / d_{\mathrm{i}}$ at $1.8 \AA$ characteristic of C.C.C contacts. Also, when the surface is mapped with shape index, several complementary triangular red hollows and blue bumps appear that are characteristic of the six-ring stacking (Fig. 4). The molecules stack in a column in a head-to-tail fashion along the $b$ axis (Fig. 5). The molecules in these stacks lie across centres of symmetry at $\left(\frac{1}{2}, 1, \frac{1}{2}\right)$, 
Table 4

Selected $\pi-\pi$ contacts.

\begin{tabular}{|c|c|c|c|c|c|c|}
\hline Compound & $C g I$ & $C g J(\operatorname{aru})$ & $C g-C g(\AA)$ & CgI_Perp (̊̊) & CgJ_Perp (§) & Slippage $(\AA)$ \\
\hline 1 & $C g 1$ & $\operatorname{Cg} 2(-x+1,-y+1,-z-1)$ & $3.911(3)$ & $-3.3848(4)$ & $-3.3352(4)$ & 2.043 \\
\hline 1 & $C g 1$ & $\operatorname{Cg} 4(-x+1,-y+1,-z-1)$ & $3.525(2)$ & $-3.3851(4)$ & $-3.2952(4)$ & 1.252 \\
\hline 1 & Cg2 & $\operatorname{Cg} 1(-x+1,-y+1,-z-1)$ & $3.911(3)$ & $-3.3352(4)$ & $-3.3849(4)$ & 1.960 \\
\hline 1 & $C g 2$ & $\operatorname{Cg} 3(-x+1,-y+1,-z-1)$ & 3.797 (3) & $-3.3389(4)$ & $-3.5276(5)$ & 1.406 \\
\hline 1 & Cg3 & $\operatorname{Cg} 2(-x+1,-y+1,-z-1)$ & $3.798(3)$ & $-3.5277(5)$ & $-3.3388(4)$ & 1.809 \\
\hline
\end{tabular}

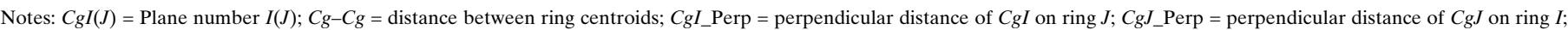

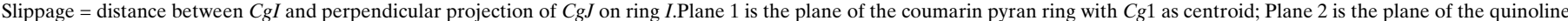

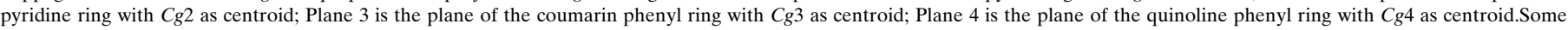
planes are repeated since they are inclined to each other and as a result give slightly different slippages

Table 5

Experimental details.

\begin{tabular}{ll}
\hline Crystal data & \\
Chemical formula & $\mathrm{C}_{20} \mathrm{H}_{14} \mathrm{~N}_{2} \mathrm{O}_{3}$ \\
$M_{\mathrm{r}}$ & 330.33 \\
Crystal system, space group & Monoclinic, $P 2_{1} / n$ \\
Temperature $(\mathrm{K})$ & 100 \\
$a, b, c(\AA)$ & $7.799(3), 7.014(3), 27.640(18)$ \\
$\beta\left({ }^{\circ}\right)$ & $90.18(6)$ \\
$V\left(\AA^{3}\right)$ & $1512.0(13)$ \\
$Z$ & 4 \\
Radiation type & Synchrotron, $\lambda=0.68891 \AA$ \\
$\mu\left(\mathrm{mm}^{-1}\right)$ & 0.09 \\
Crystal size $(\mathrm{mm})$ & $0.18 \times 0.01 \times 0.004$ \\
& \\
Data collection & \\
Diffractometer & Three-circle diffractometer \\
Absorption correction & Empirical (using intensity \\
& measurements) (aimless $C C P 4 ;$ \\
No. of measured, independent and & Evans, 2006) \\
$\quad$ observed $[I>2 \sigma(I)]$ reflections & $18408,4587,3717$ \\
$R_{\text {int }}$ & \\
$(\text { sin } \theta / \lambda)_{\text {max }}\left(\AA^{-1}\right)$ & 0.060 \\
& 0.714 \\
Refinement & \\
$R\left[F^{2}>2 \sigma\left(F^{2}\right)\right], w R\left(F^{2}\right), S$ & \\
No. of reflections & \\
No. of parameters & $0.051,0.156,1.13$ \\
H-atom treatment & 4587 \\
& 231 \\
$\Delta \rho_{\text {max }}, \Delta \rho_{\text {min }}\left(\mathrm{e} \AA \AA^{-3}\right)$ & $\mathrm{H}$ atoms treated by a mixture of \\
\hline
\end{tabular}

Computer programs: GDA http://www.opengda.org/OpenGDA.html, XIA2 0.4.0.370g47f3bc3 (Winter, 2010), SHELXT (Sheldrick, 2015a), ShelXle (Hübschle et al., 2011), SHELXL2014 (Sheldrick, 2015b), Mercury (Macrae et al., 2006) and PLATON (Spek, 2009).

a centrosymetrically related contact between the pyran and pyridine rings, and across the centre at $\left(\frac{1}{2}, \frac{1}{2}, \frac{1}{2}\right)$, which involves three short centrosymmetrically related contacts: (i) between the pyran and pyridine rings, (ii) between the pyran ring and the quinoline phenyl ring and (iii) between the coumarin phenyl ring and the pyridine ring.

\section{Database survey}

As reported by Gomes et al. (2016), a search made in the Cambridge Structural Database (CSD, Version 35.7; Groom et

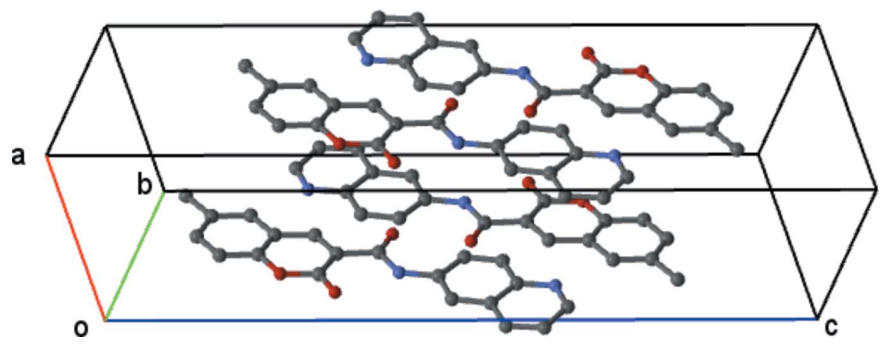

Figure 5

View of the $\pi-\pi$ stacking along the $b$ axis.

al., 2016) revealed the existence of 35 deposited compounds (42 molecules) containing the coumarin carboxamide unit, all of which contained the same intramolecular hydrogen bonds. The present compound also contains these bonds, as described above.

\section{Synthesis and crystallization}

6-Methylcoumarin-3-carboxylic acid (Murata et al.., 2005) (1 mmol) was dissolved in dichloromethane and 3-[3-(dimethylamino)propyl]-1-ethylcarbodiimide $(1.10 \mathrm{mmol})$ and 4-dimethylaminopyridine $(1.10 \mathrm{mmol})$ were added. The mixture was kept under a flux of argon at $273 \mathrm{~K}$ for $5 \mathrm{~min}$. 6-Aminoquinoline $(1 \mathrm{mmol})$ was then added in small portions. The reaction mixture was stirred for $4 \mathrm{~h}$ at room temperature. The obtained precipitate was filtered off and recrystallized from methanol to give colourless needles of (1). Overall yield: 53\%; m.p. 545-546 K.

\section{Refinement}

$\mathrm{H}$ atoms were treated as riding atoms, with aromatic $\mathrm{C}-\mathrm{H}=$ $0.95 \AA$, with $U_{\text {iso }}(\mathrm{H})=1.2 U_{\text {eq }}(\mathrm{C})$, and methyl $\mathrm{C}-\mathrm{H}=0.98 \AA$, with $U_{\text {iso }}(\mathrm{H})=1.5 U_{\text {eq }}(\mathrm{C})$. The amino $\mathrm{H}$ atoms were freely refined. Crystal data, data collection and structure refinement details are summarized in Table 5. 


\section{Acknowledgements}

The authors thank the staff at the National Crystallographic Service, University of Southampton, for the data collection, help and advice (Coles \& Gale, 2012), and the Foundation for Science and Technology (FCT) and FEDER/COMPETE2020 (UID/QU $\iota 00081 / 2015$ and POCI-01-0145-FEDER-006980). AF (SFRH/BD/80831/2011) and MJM (SFRH/BPD/95345/ 2013) were supported by grants from FCT, POPH and QREN.

\section{References}

Camps, P., Formosa, X., Galdeano, C., Muñoz-Torrero, D., Ramírez, L., Gómez, E., Isambert, N., Lavilla, R., Badia, A., Clos, M. V., Bartolini, M., Mancini, F., Andrisano, V., Arce, M. P., RodríguezFranco, M. I., Huertas, O., Dafni, T. \& Luque, F. J. (2009). J. Med. Chem. 52, 5365-5379.

Chimenti, F., Bizzarri, B., Bolasco, A., Secci, D., Chimenti, P., Granese, A., Carradori, S., Rivanera, D., Zicari, A., Scaltrito, M. M. \& Sisto, F. (2010). Bioorg. Med. Chem. Lett. 20, 4922-4926.

Coles, S. J. \& Gale, P. A. (2012). Chem. Sci. 3, 683-689.

Evans, P. (2006). Acta Cryst. D62, 72-82.

Gomes, L. R., Low, J. N., Fonseca, A., Matos, M. J. \& Borges, F. (2016). Acta Cryst. E72, 926-932.

Groom, C. R., Bruno, I. J., Lightfoot, M. P. \& Ward, S. C. (2016). Acta Cryst. B72, 171-179.
Hübschle, C. B., Sheldrick, G. M. \& Dittrich, B. (2011). J. Appl. Cryst. 44, 1281-1284.

Macrae, C. F., Edgington, P. R., McCabe, P., Pidcock, E., Shields, G. P., Taylor, R., Towler, M. \& van de Streek, J. (2006). J. Appl. Cryst. 39, 453-457.

Matos, M. J., Janeiro, P., Gonzl'alez Franco, R. M., Vilar, S., Tatonetti, N. P., Santana, L., Uriarte, E., Borges, F., Fontenla, J. A. \& Viña, D. (2014). Future Med. Chem. 6, 371-383.

Matos, M. J., Viña, D., Quezada, E., Picciau, C., Delogu, G., Orallo, F., Santana, L. \& Uriarte, E. (2009). Bioorg. Med. Chem. Lett. 19, 3268-3270.

Murata, C., Masuda, T., Kamochi, Y., Todoroki, K., Yoshida, H., Nohta, H., Yamaguchi, M. \& Takadate, A. (2005). Chem. Pharm. Bull. (Tokyo), 53, 750-758.

Rohl, A. L., Moret, M., Kaminsky, W., Claborn, K., McKinnon, J. J. \& Kahr, B. (2008). Cryst. Growth Des. 8, 451-4525.

Sheldrick, G. M. (2015a). Acta Cryst. A71, 3-8.

Sheldrick, G. M. (2015b). Acta Cryst. C71, 3-8.

Spek, A. L. (2009). Acta Cryst. D65, 148-155.

Sridharan, V., Suryavanshi, P. \& Menéndez, J. C. (2011). Chem. Rev. 111, 7157-7259.

Vazquez-Rodriguez, S., Matos, M. J., Santana, L., Uriarte, E., Borges, F., Kachler, S. \& Klotz, K. N. (2013). J. Pharm. Pharmacol. 65, 607703.

Winter, G. (2010). J. Appl. Cryst. 43, 186-190.

Wolff, S. K., Grimwood, D. J., McKinnon, J. J., Turner, M. J., Jayatilaka, D. \& Spackman, M. A. (2012). Crystal Explorer. The University of Western Australia. 


\section{supporting information}

Acta Cryst. (2016). E72, 1121-1125 [https://doi.org/10.1107/S2056989016011026]

\section{6-Methyl-2-oxo- $\mathrm{N}$-(quinolin-6-yl)-2H-chromene-3-carboxamide: crystal structure and Hirshfeld surface analysis}

\section{Lígia R. Gomes, John Nicolson Low, André Fonseca, Maria João Matos and Fernanda Borges}

\section{Computing details}

Data collection: GDA http://www.opengda.org/OpenGDA.html; cell refinement: XIA2 0.4.0.370-g47f3bc3, (Winter, 2010; data reduction: XIA2 0.4.0.370-g47f3bc3 (Winter, 2010; program(s) used to solve structure: SHELXT (Sheldrick, 2015a); program(s) used to refine structure: ShelXle (Hübschle et al., 2011) SHELXL2014/7 (Sheldrick, 2015b); molecular graphics: Mercury (Macrae et al., 2006); software used to prepare material for publication: SHELXL2014/17 (Sheldrick, 2015b) PLATON (Spek, 2009).

(I)

Crystal data

$\mathrm{C}_{20} \mathrm{H}_{14} \mathrm{~N}_{2} \mathrm{O}_{3}$

$M_{r}=330.33$

Monoclinic, $P 2_{1} / n$

$a=7.799(3) \AA$

$b=7.014(3) \AA$

$c=27.640(18) \AA$

$\beta=90.18(6)^{\circ}$

$V=1512.0(13) \AA^{3}$

$Z=4$

Data collection

Three-circle diffractometer

Radiation source: synchrotron, DLS beamline I19, undulator

Si 111, double crystal monochromator Detector resolution: 5.81 pixels $\mathrm{mm}^{-1}$ profile data from $\omega$-scans

Absorption correction: empirical (using intensity measurements) aimless ccp4 (Evans, 2006)

Refinement

Refinement on $F^{2}$

Least-squares matrix: full

$R\left[F^{2}>2 \sigma\left(F^{2}\right)\right]=0.051$

$w R\left(F^{2}\right)=0.156$

$S=1.13$

4587 reflections
$F(000)=688$

$D_{\mathrm{x}}=1.451 \mathrm{Mg} \mathrm{m}^{-3}$

Synchrotron' radiation, $\lambda=0.68891 \AA$

Cell parameters from 3773 reflections

$\theta=2.6-33.9^{\circ}$

$\mu=0.09 \mathrm{~mm}^{-1}$

$T=100 \mathrm{~K}$

Needle, colourless

$0.18 \times 0.01 \times 0.004 \mathrm{~mm}$

18408 measured reflections

4587 independent reflections

3717 reflections with $I>2 \sigma(I)$

$R_{\text {int }}=0.060$

$\theta_{\max }=29.5^{\circ}, \theta_{\min }=2.9^{\circ}$

$h=-11 \rightarrow 11$

$k=-10 \rightarrow 10$

$l=-39 \rightarrow 39$

\section{1 parameters}

0 restraints

Hydrogen site location: mixed

$\mathrm{H}$ atoms treated by a mixture of independent and constrained refinement 
$w=1 /\left[\sigma^{2}\left(F_{\mathrm{o}}^{2}\right)+(0.0954 P)^{2}\right]$

where $P=\left(F_{\mathrm{o}}^{2}+2 F_{\mathrm{c}}^{2}\right) / 3$

$(\Delta / \sigma)_{\max }=0.001$

$$
\Delta \rho_{\max }=0.54 \mathrm{e} \AA^{-3}
$$

$\Delta \rho_{\min }=-0.25$ e $\AA^{-3}$

\section{Special details}

Geometry. All esds (except the esd in the dihedral angle between two 1.s. planes) are estimated using the full covariance matrix. The cell esds are taken into account individually in the estimation of esds in distances, angles and torsion angles; correlations between esds in cell parameters are only used when they are defined by crystal symmetry. An approximate (isotropic) treatment of cell esds is used for estimating esds involving l.s. planes.

Fractional atomic coordinates and isotropic or equivalent isotropic displacement parameters $\left(\hat{A}^{2}\right)$

\begin{tabular}{|c|c|c|c|c|}
\hline & $x$ & $y$ & $z$ & $U_{\text {iso }} * / U_{\text {eq }}$ \\
\hline $\mathrm{O} 1$ & $0.61633(10)$ & $0.57511(12)$ & $0.33247(3)$ & 0.02193 (19) \\
\hline $\mathrm{O} 2$ & $0.42390(10)$ & $0.67655(12)$ & $0.38475(3)$ & $0.0244(2)$ \\
\hline O31 & $0.79011(10)$ & $0.65810(12)$ & $0.49728(3)$ & $0.0240(2)$ \\
\hline N32 & $0.50968(12)$ & $0.71842(13)$ & $0.47835(3)$ & $0.0186(2)$ \\
\hline N311 & $0.21868(12)$ & $0.98576(13)$ & $0.65151(3)$ & $0.0204(2)$ \\
\hline $\mathrm{C} 2$ & $0.57274(14)$ & $0.62613(16)$ & 0.37867 (4) & $0.0196(2)$ \\
\hline $\mathrm{C} 3$ & $0.70619(13)$ & $0.61438(15)$ & $0.41571(4)$ & $0.0177(2)$ \\
\hline $\mathrm{C} 4$ & $0.86585(13)$ & $0.55411(15)$ & $0.40316(4)$ & $0.0180(2)$ \\
\hline H4 & 0.9519 & 0.5451 & 0.4275 & $0.022 *$ \\
\hline $\mathrm{C} 5$ & $1.07250(14)$ & $0.44450(15)$ & $0.33910(4)$ & $0.0200(2)$ \\
\hline H5 & 1.1628 & 0.4362 & 0.3622 & $0.024 *$ \\
\hline $\mathrm{C} 4 \mathrm{~A}$ & $0.90818(14)$ & $0.50369(15)$ & $0.35425(4)$ & $0.0182(2)$ \\
\hline C6 & $1.10480(15)$ & $0.39806(16)$ & $0.29103(4)$ & $0.0222(2)$ \\
\hline $\mathrm{C} 7$ & $0.97020(15)$ & $0.41362(17)$ & $0.25763(4)$ & $0.0238(2)$ \\
\hline $\mathrm{H} 7$ & 0.9904 & 0.3821 & 0.2247 & $0.029 *$ \\
\hline $\mathrm{C} 8$ & $0.80789(15)$ & $0.47398(17)$ & $0.27134(4)$ & $0.0230(2)$ \\
\hline H8 & 0.7184 & 0.4858 & 0.2482 & $0.028 *$ \\
\hline C8A & $0.77937(14)$ & $0.51669(16)$ & $0.31972(4)$ & $0.0195(2)$ \\
\hline C31 & $0.67345(13)$ & $0.66578(15)$ & $0.46783(4)$ & $0.0185(2)$ \\
\hline C34A & $0.18814(13)$ & $0.89163(15)$ & $0.56639(4)$ & $0.0176(2)$ \\
\hline C38A & $0.28705(13)$ & $0.91692(15)$ & $0.60890(4)$ & $0.0178(2)$ \\
\hline C61 & $1.27981(16)$ & $0.3329(2)$ & $0.27477(5)$ & $0.0312(3)$ \\
\hline H61A & 1.3103 & 0.2147 & 0.2916 & $0.047 *$ \\
\hline H61B & 1.3647 & 0.4316 & 0.2824 & $0.047 *$ \\
\hline H61C & 1.2781 & 0.3101 & 0.2398 & $0.047 *$ \\
\hline C312 & $0.05500(14)$ & $1.03063(16)$ & $0.65149(4)$ & 0.0219 \\
\hline H312 & 0.0075 & 1.0795 & 0.6806 & $0.026^{*}$ \\
\hline C313 & $-0.05445(14)$ & $1.01098(16)$ & $0.61122(4)$ & $0.0219(2)$ \\
\hline H313 & -0.1717 & 1.0466 & 0.6133 & $0.026^{*}$ \\
\hline C314 & $0.01123(13)$ & $0.93934(16)$ & $0.56876(4)$ & $0.0199(2)$ \\
\hline H314 & -0.0609 & 0.9220 & 0.5413 & $0.024 *$ \\
\hline C315 & $0.26757(13)$ & $0.82186(15)$ & 0.52375 & $0.0183(2)$ \\
\hline H315 & 0.2006 & 0.8037 & 0.4954 & $0.022 *$ \\
\hline C316 & $0.44016(14)$ & $0.78004(15)$ & $0.52286(4)$ & $0.0178(2)$ \\
\hline C317 & $0.53856(14)$ & $0.80040(16)$ & 0.56555 & $0.0197(2)$ \\
\hline H317 & 0.6569 & 0.7683 & 0.5655 & $0.024 *$ \\
\hline
\end{tabular}




\begin{tabular}{lllll}
$\mathrm{C} 318$ & $0.46224(14)$ & $0.86712(16)$ & $0.60735(4)$ & $0.0198(2)$ \\
$\mathrm{H} 318$ & 0.5297 & 0.8798 & 0.6358 & $0.024^{*}$ \\
$\mathrm{H} 32$ & $0.440(2)$ & $0.709(3)$ & $0.4521(6)$ & $0.042(5)^{*}$ \\
\hline
\end{tabular}

Atomic displacement parameters $\left(\AA^{2}\right)$

\begin{tabular}{lllllll}
\hline & $U^{11}$ & $U^{22}$ & $U^{33}$ & $U^{12}$ & $U^{13}$ & $U^{23}$ \\
\hline O1 & $0.0188(4)$ & $0.0271(4)$ & $0.0199(4)$ & $-0.0001(3)$ & $0.0003(3)$ & $-0.0025(3)$ \\
O2 & $0.0186(4)$ & $0.0295(5)$ & $0.0250(4)$ & $0.0018(3)$ & $-0.0007(3)$ & $-0.0027(3)$ \\
O31 & $0.0209(4)$ & $0.0295(5)$ & $0.0216(4)$ & $0.0024(3)$ & $-0.0005(3)$ & $-0.0043(3)$ \\
N32 & $0.0184(4)$ & $0.0194(5)$ & $0.0180(4)$ & $0.0012(3)$ & $0.0018(3)$ & $-0.0009(3)$ \\
N311 & $0.0226(4)$ & $0.0201(5)$ & $0.0186(5)$ & $-0.0011(3)$ & $0.0019(3)$ & $-0.0011(3)$ \\
C2 & $0.0200(5)$ & $0.0186(5)$ & $0.0203(5)$ & $-0.0020(4)$ & $0.0014(4)$ & $-0.0007(4)$ \\
C3 & $0.0185(5)$ & $0.0164(5)$ & $0.0183(5)$ & $-0.0012(4)$ & $0.0012(4)$ & $-0.0004(4)$ \\
C4 & $0.0192(5)$ & $0.0155(5)$ & $0.0193(5)$ & $-0.0012(4)$ & $0.0008(4)$ & $0.0000(4)$ \\
C5 & $0.0208(5)$ & $0.0178(5)$ & $0.0214(5)$ & $-0.0001(4)$ & $0.0019(4)$ & $-0.0001(4)$ \\
C4A & $0.0199(5)$ & $0.0154(5)$ & $0.0194(5)$ & $-0.0022(4)$ & $0.0021(4)$ & $-0.0006(4)$ \\
C6 & $0.0242(5)$ & $0.0197(5)$ & $0.0227(5)$ & $-0.0012(4)$ & $0.0046(4)$ & $-0.0018(4)$ \\
C7 & $0.0273(5)$ & $0.0240(6)$ & $0.0202(5)$ & $-0.0030(4)$ & $0.0042(4)$ & $-0.0024(4)$ \\
C8 & $0.0248(5)$ & $0.0258(6)$ & $0.0185(5)$ & $-0.0030(4)$ & $-0.0011(4)$ & $-0.0013(4)$ \\
C8A & $0.0188(5)$ & $0.0193(5)$ & $0.0202(5)$ & $-0.0024(4)$ & $0.0021(4)$ & $-0.0007(4)$ \\
C31 & $0.0194(5)$ & $0.0151(5)$ & $0.0212(5)$ & $-0.0009(4)$ & $0.0025(4)$ & $-0.0005(4)$ \\
C34A & $0.0178(5)$ & $0.0144(5)$ & $0.0205(5)$ & $-0.0002(3)$ & $0.0016(4)$ & $0.0011(4)$ \\
C38A & $0.0194(5)$ & $0.0158(5)$ & $0.0181(5)$ & $-0.0013(4)$ & $0.0016(4)$ & $0.0003(4)$ \\
C61 & $0.0253(6)$ & $0.0408(8)$ & $0.0274(6)$ & $0.0069(5)$ & $0.0052(5)$ & $-0.0060(5)$ \\
C312 & $0.0238(5)$ & $0.0204(5)$ & $0.0214(5)$ & $-0.0012(4)$ & $0.0057(4)$ & $-0.0012(4)$ \\
C313 & $0.0188(5)$ & $0.0216(5)$ & $0.0251(6)$ & $0.0002(4)$ & $0.0032(4)$ & $0.0006(4)$ \\
C314 & $0.0180(5)$ & $0.0202(5)$ & $0.0215(5)$ & $-0.0006(4)$ & $-0.0001(4)$ & $0.0015(4)$ \\
C315 & $0.0195(5)$ & $0.0167(5)$ & $0.0188(5)$ & $0.0004(4)$ & $-0.0001(4)$ & $0.0003(4)$ \\
C316 & $0.0201(5)$ & $0.0148(5)$ & $0.0185(5)$ & $-0.0001(4)$ & $0.0029(4)$ & $0.0006(4)$ \\
C317 & $0.0183(5)$ & $0.0203(5)$ & $0.0206(5)$ & $0.0010(4)$ & $0.0016(4)$ & $0.0002(4)$ \\
C318 & $0.0204(5)$ & $0.0207(5)$ & $0.0185(5)$ & $-0.0005(4)$ & $-0.0011(4)$ & $-0.0002(4)$ \\
& & & & & &
\end{tabular}

Geometric parameters $(\AA, \stackrel{o}{)})$

\begin{tabular}{llll}
\hline $\mathrm{O} 1-\mathrm{C} 2$ & $1.3701(16)$ & $\mathrm{C} 7-\mathrm{H} 7$ & 0.9500 \\
$\mathrm{O} 1-\mathrm{C} 8 \mathrm{~A}$ & $1.3827(14)$ & $\mathrm{C} 8-\mathrm{C} 8 \mathrm{~A}$ & $1.3887(18)$ \\
$\mathrm{O} 2-\mathrm{C} 2$ & $1.2255(14)$ & $\mathrm{C} 8-\mathrm{H} 8$ & 0.9500 \\
$\mathrm{O} 31-\mathrm{C} 31$ & $1.2201(16)$ & $\mathrm{C} 34 \mathrm{~A}-\mathrm{C} 38 \mathrm{~A}$ & $1.4149(18)$ \\
$\mathrm{N} 32-\mathrm{C} 31$ & $1.3618(14)$ & $\mathrm{C} 34 \mathrm{~A}-\mathrm{C} 315$ & $1.4200(17)$ \\
$\mathrm{N} 32-\mathrm{C} 316$ & $1.4136(16)$ & $\mathrm{C} 34 \mathrm{~A}-\mathrm{C} 314$ & $1.4214(15)$ \\
$\mathrm{N} 32-\mathrm{H} 32$ & $0.907(18)$ & $\mathrm{C} 38 \mathrm{~A}-\mathrm{C} 318$ & $1.4111(16)$ \\
$\mathrm{N} 311-\mathrm{C} 312$ & $1.3147(15)$ & $\mathrm{C} 61-\mathrm{H} 61 \mathrm{~A}$ & 0.9800 \\
$\mathrm{~N} 311-\mathrm{C} 38 \mathrm{~A}$ & $1.3814(16)$ & $\mathrm{C} 61-\mathrm{H} 61 \mathrm{~B}$ & 0.9800 \\
$\mathrm{C} 2-\mathrm{C} 3$ & $1.4600(18)$ & $\mathrm{C} 61-\mathrm{H} 61 \mathrm{C}$ & 0.9800 \\
$\mathrm{C} 3-\mathrm{C} 4$ & $1.3609(15)$ & $\mathrm{C} 312-\mathrm{C} 313$ & $1.4075(19)$ \\
$\mathrm{C} 3-\mathrm{C} 31$ & $1.5075(18)$ & $\mathrm{C} 312-\mathrm{H} 312$ & 0.9500 \\
$\mathrm{C} 4-\mathrm{C} 4 \mathrm{~A}$ & $1.4368(17)$ & $\mathrm{C} 313-\mathrm{C} 314$ & $1.3769(17)$
\end{tabular}




\begin{tabular}{|c|c|c|c|}
\hline $\mathrm{C} 4-\mathrm{H} 4$ & 0.9500 & C313-H313 & 0.9500 \\
\hline $\mathrm{C} 5-\mathrm{C} 6$ & $1.3918(18)$ & C314-H314 & 0.9500 \\
\hline $\mathrm{C} 5-\mathrm{C} 4 \mathrm{~A}$ & $1.4119(16)$ & $\mathrm{C} 315-\mathrm{C} 316$ & $1.3779(15)$ \\
\hline $\mathrm{C} 5-\mathrm{H} 5$ & 0.9500 & C315-H315 & 0.9500 \\
\hline $\mathrm{C} 4 \mathrm{~A}-\mathrm{C} 8 \mathrm{~A}$ & $1.3866(18)$ & C316-C317 & $1.4128(18)$ \\
\hline $\mathrm{C} 6-\mathrm{C} 7$ & $1.4000(19)$ & C317-C318 & $1.3830(17)$ \\
\hline C6-C61 & $1.5092(17)$ & C317- 3317 & 0.9500 \\
\hline $\mathrm{C} 7-\mathrm{C} 8$ & $1.3886(17)$ & C318-H318 & 0.9500 \\
\hline $\mathrm{C} 2-\mathrm{O} 1-\mathrm{C} 8 \mathrm{~A}$ & $123.10(10)$ & $\mathrm{N} 32-\mathrm{C} 31-\mathrm{C} 3$ & $115.51(11)$ \\
\hline $\mathrm{C} 31-\mathrm{N} 32-\mathrm{C} 316$ & $129.15(11)$ & $\mathrm{C} 38 \mathrm{~A}-\mathrm{C} 34 \mathrm{~A}-\mathrm{C} 315$ & $119.63(10)$ \\
\hline $\mathrm{C} 31-\mathrm{N} 32-\mathrm{H} 32$ & $111.5(11)$ & $\mathrm{C} 38 \mathrm{~A}-\mathrm{C} 34 \mathrm{~A}-\mathrm{C} 314$ & $117.32(10)$ \\
\hline $\mathrm{C} 316-\mathrm{N} 32-\mathrm{H} 32$ & $119.3(11)$ & $\mathrm{C} 315-\mathrm{C} 34 \mathrm{~A}-\mathrm{C} 314$ & $123.05(11)$ \\
\hline $\mathrm{C} 312-\mathrm{N} 311-\mathrm{C} 38 \mathrm{~A}$ & $117.43(11)$ & $\mathrm{N} 311-\mathrm{C} 38 \mathrm{~A}-\mathrm{C} 318$ & $119.26(11)$ \\
\hline $\mathrm{O} 2-\mathrm{C} 2-\mathrm{O} 1$ & $116.17(11)$ & $\mathrm{N} 311-\mathrm{C} 38 \mathrm{~A}-\mathrm{C} 34 \mathrm{~A}$ & $122.75(10)$ \\
\hline $\mathrm{O} 2-\mathrm{C} 2-\mathrm{C} 3$ & $126.42(11)$ & $\mathrm{C} 318-\mathrm{C} 38 \mathrm{~A}-\mathrm{C} 34 \mathrm{~A}$ & $117.98(10)$ \\
\hline $\mathrm{O} 1-\mathrm{C} 2-\mathrm{C} 3$ & $117.42(10)$ & C6-C61-H61A & 109.5 \\
\hline $\mathrm{C} 4-\mathrm{C} 3-\mathrm{C} 2$ & $119.32(11)$ & C6- $\mathrm{C} 61-\mathrm{H} 61 \mathrm{~B}$ & 109.5 \\
\hline $\mathrm{C} 4-\mathrm{C} 3-\mathrm{C} 31$ & $118.40(11)$ & $\mathrm{H} 61 \mathrm{~A}-\mathrm{C} 61-\mathrm{H} 61 \mathrm{~B}$ & 109.5 \\
\hline $\mathrm{C} 2-\mathrm{C} 3-\mathrm{C} 31$ & $122.28(10)$ & $\mathrm{C} 6-\mathrm{C} 61-\mathrm{H} 61 \mathrm{C}$ & 109.5 \\
\hline $\mathrm{C} 3-\mathrm{C} 4-\mathrm{C} 4 \mathrm{~A}$ & $121.96(11)$ & $\mathrm{H} 61 \mathrm{~A}-\mathrm{C} 61-\mathrm{H} 61 \mathrm{C}$ & 109.5 \\
\hline $\mathrm{C} 3-\mathrm{C} 4-\mathrm{H} 4$ & 119.0 & $\mathrm{H} 61 \mathrm{~B}-\mathrm{C} 61-\mathrm{H} 61 \mathrm{C}$ & 109.5 \\
\hline $\mathrm{C} 4 \mathrm{~A}-\mathrm{C} 4-\mathrm{H} 4$ & 119.0 & $\mathrm{~N} 311-\mathrm{C} 312-\mathrm{C} 313$ & $124.28(11)$ \\
\hline $\mathrm{C} 6-\mathrm{C} 5-\mathrm{C} 4 \mathrm{~A}$ & $121.27(12)$ & $\mathrm{N} 311-\mathrm{C} 312-\mathrm{H} 312$ & 117.9 \\
\hline $\mathrm{C} 6-\mathrm{C} 5-\mathrm{H} 5$ & 119.4 & $\mathrm{C} 313-\mathrm{C} 312-\mathrm{H} 312$ & 117.9 \\
\hline $\mathrm{C} 4 \mathrm{~A}-\mathrm{C} 5-\mathrm{H} 5$ & 119.4 & $\mathrm{C} 314-\mathrm{C} 313-\mathrm{C} 312$ & $118.92(10)$ \\
\hline $\mathrm{C} 8 \mathrm{~A}-\mathrm{C} 4 \mathrm{~A}-\mathrm{C} 5$ & $118.13(11)$ & $\mathrm{C} 314-\mathrm{C} 313-\mathrm{H} 313$ & 120.5 \\
\hline $\mathrm{C} 8 \mathrm{~A}-\mathrm{C} 4 \mathrm{~A}-\mathrm{C} 4$ & $117.61(11)$ & $\mathrm{C} 312-\mathrm{C} 313-\mathrm{H} 313$ & 120.5 \\
\hline $\mathrm{C} 5-\mathrm{C} 4 \mathrm{~A}-\mathrm{C} 4$ & $124.25(11)$ & $\mathrm{C} 313-\mathrm{C} 314-\mathrm{C} 34 \mathrm{~A}$ & $119.27(11)$ \\
\hline $\mathrm{C} 5-\mathrm{C} 6-\mathrm{C} 7$ & $118.28(11)$ & $\mathrm{C} 313-\mathrm{C} 314-\mathrm{H} 314$ & 120.4 \\
\hline $\mathrm{C} 5-\mathrm{C} 6-\mathrm{C} 61$ & $121.45(12)$ & $\mathrm{C} 34 \mathrm{~A}-\mathrm{C} 314-\mathrm{H} 314$ & 120.4 \\
\hline $\mathrm{C} 7-\mathrm{C} 6-\mathrm{C} 61$ & $120.28(11)$ & $\mathrm{C} 316-\mathrm{C} 315-\mathrm{C} 34 \mathrm{~A}$ & $121.13(11)$ \\
\hline $\mathrm{C} 8-\mathrm{C} 7-\mathrm{C} 6$ & $121.73(11)$ & C $316-\mathrm{C} 315-\mathrm{H} 315$ & 119.4 \\
\hline $\mathrm{C} 8-\mathrm{C} 7-\mathrm{H} 7$ & 119.1 & $\mathrm{C} 34 \mathrm{~A}-\mathrm{C} 315-\mathrm{H} 315$ & 119.4 \\
\hline $\mathrm{C} 6-\mathrm{C} 7-\mathrm{H} 7$ & 119.1 & $\mathrm{C} 315-\mathrm{C} 316-\mathrm{C} 317$ & $119.45(11)$ \\
\hline $\mathrm{C} 7-\mathrm{C} 8-\mathrm{C} 8 \mathrm{~A}$ & $118.52(12)$ & $\mathrm{C} 315-\mathrm{C} 316-\mathrm{N} 32$ & $117.26(11)$ \\
\hline $\mathrm{C} 7-\mathrm{C} 8-\mathrm{H} 8$ & 120.7 & $\mathrm{C} 317-\mathrm{C} 316-\mathrm{N} 32$ & $123.29(10)$ \\
\hline $\mathrm{C} 8 \mathrm{~A}-\mathrm{C} 8-\mathrm{H} 8$ & 120.7 & C318-C317-C316 & $119.86(10)$ \\
\hline $\mathrm{O} 1-\mathrm{C} 8 \mathrm{~A}-\mathrm{C} 4 \mathrm{~A}$ & $120.59(10)$ & C318-C317-H317 & 120.1 \\
\hline $\mathrm{O} 1-\mathrm{C} 8 \mathrm{~A}-\mathrm{C} 8$ & $117.36(11)$ & C316-C317-H317 & 120.1 \\
\hline $\mathrm{C} 4 \mathrm{~A}-\mathrm{C} 8 \mathrm{~A}-\mathrm{C} 8$ & $122.06(11)$ & $\mathrm{C} 317-\mathrm{C} 318-\mathrm{C} 38 \mathrm{~A}$ & $121.90(11)$ \\
\hline $\mathrm{O} 31-\mathrm{C} 31-\mathrm{N} 32$ & $124.57(11)$ & C317-C $318-\mathrm{H} 318$ & 119.0 \\
\hline $\mathrm{O} 31-\mathrm{C} 31-\mathrm{C} 3$ & $119.91(10)$ & $\mathrm{C} 38 \mathrm{~A}-\mathrm{C} 318-\mathrm{H} 318$ & 119.0 \\
\hline $\mathrm{C} 8 \mathrm{~A}-\mathrm{O} 1-\mathrm{C} 2-\mathrm{O} 2$ & $179.41(9)$ & $\mathrm{C} 4-\mathrm{C} 3-\mathrm{C} 31-\mathrm{O} 31$ & $2.12(16)$ \\
\hline $\mathrm{C} 8 \mathrm{~A}-\mathrm{O} 1-\mathrm{C} 2-\mathrm{C} 3$ & $-0.94(15)$ & $\mathrm{C} 2-\mathrm{C} 3-\mathrm{C} 31-\mathrm{O} 31$ & $-178.23(10)$ \\
\hline $\mathrm{O} 2-\mathrm{C} 2-\mathrm{C} 3-\mathrm{C} 4$ & $179.54(11)$ & $\mathrm{C} 4-\mathrm{C} 3-\mathrm{C} 31-\mathrm{N} 32$ & $-177.74(9)$ \\
\hline $\mathrm{O} 1-\mathrm{C} 2-\mathrm{C} 3-\mathrm{C} 4$ & $-0.06(15)$ & $\mathrm{C} 2-\mathrm{C} 3-\mathrm{C} 31-\mathrm{N} 32$ & $1.91(15)$ \\
\hline
\end{tabular}




$\begin{array}{llll}\mathrm{O} 2-\mathrm{C} 2-\mathrm{C} 3-\mathrm{C} 31 & -0.09(18) & \mathrm{C} 312-\mathrm{N} 311-\mathrm{C} 38 \mathrm{~A}-\mathrm{C} 318 & 179.98(10) \\ \mathrm{O} 1-\mathrm{C} 2-\mathrm{C} 3-\mathrm{C} 31 & -179.70(9) & \mathrm{C} 312-\mathrm{N} 311-\mathrm{C} 38 \mathrm{~A}-\mathrm{C} 34 \mathrm{~A} & -0.77(16) \\ \mathrm{C} 2-\mathrm{C} 3-\mathrm{C} 4-\mathrm{C} 4 \mathrm{~A} & 0.81(16) & \mathrm{C} 315-\mathrm{C} 34 \mathrm{~A}-\mathrm{C} 38 \mathrm{~A}-\mathrm{N} 311 & 179.40(10) \\ \mathrm{C} 31-\mathrm{C} 3-\mathrm{C} 4-\mathrm{C} 4 \mathrm{~A} & -179.54(9) & \mathrm{C} 314-\mathrm{C} 34 \mathrm{~A}-\mathrm{C} 38 \mathrm{~A}-\mathrm{N} 311 & -0.32(16) \\ \mathrm{C} 6-\mathrm{C} 5-\mathrm{C} 4 \mathrm{~A}-\mathrm{C} 8 \mathrm{~A} & -0.76(16) & \mathrm{C} 315-\mathrm{C} 34 \mathrm{~A}-\mathrm{C} 38 \mathrm{~A}-\mathrm{C} 318 & -1.34(15) \\ \mathrm{C} 6-\mathrm{C} 5-\mathrm{C} 4 \mathrm{~A}-\mathrm{C} 4 & -179.86(10) & \mathrm{C} 314-\mathrm{C} 34 \mathrm{~A}-\mathrm{C} 38 \mathrm{~A}-\mathrm{C} 318 & 178.94(10) \\ \mathrm{C} 3-\mathrm{C} 4-\mathrm{C} 4 \mathrm{~A}-\mathrm{C} 8 \mathrm{~A} & -0.58(16) & \mathrm{C} 38 \mathrm{~A}-\mathrm{N} 311-\mathrm{C} 312-\mathrm{C} 313 & 0.74(17) \\ \mathrm{C} 3-\mathrm{C} 4-\mathrm{C} 4 \mathrm{~A}-\mathrm{C} 5 & 178.52(10) & \mathrm{N} 311-\mathrm{C} 312-\mathrm{C} 313-\mathrm{C} 314 & 0.39(18) \\ \mathrm{C} 4 \mathrm{~A}-\mathrm{C} 5-\mathrm{C} 6-\mathrm{C} 7 & 0.76(17) & \mathrm{C} 312-\mathrm{C} 313-\mathrm{C} 314-\mathrm{C} 34 \mathrm{~A} & -1.51(16) \\ \mathrm{C} 4 \mathrm{~A}-\mathrm{C} 5-\mathrm{C} 6-\mathrm{C} 61 & -179.40(11) & \mathrm{C} 38 \mathrm{~A}-\mathrm{C} 34 \mathrm{~A}-\mathrm{C} 314-\mathrm{C} 313 & 1.45(15) \\ \mathrm{C} 5-\mathrm{C} 6-\mathrm{C} 7-\mathrm{C} 8 & 0.15(18) & \mathrm{C} 315-\mathrm{C} 34 \mathrm{~A}-\mathrm{C} 314-\mathrm{C} 313 & -178.25(10) \\ \mathrm{C} 61-\mathrm{C} 6-\mathrm{C} 7-\mathrm{C} 8 & -179.69(11) & \mathrm{C} 38 \mathrm{~A}-\mathrm{C} 34 \mathrm{~A}-\mathrm{C} 315-\mathrm{C} 316 & -0.68(16) \\ \mathrm{C} 6-\mathrm{C} 7-\mathrm{C} 8-\mathrm{C} 8 \mathrm{~A} & -1.03(18) & \mathrm{C} 314-\mathrm{C} 34 \mathrm{~A}-\mathrm{C} 315-\mathrm{C} 316 & 179.02(10) \\ \mathrm{C} 2-\mathrm{O} 1-\mathrm{C} 8 \mathrm{~A}-\mathrm{C} 4 \mathrm{~A} & 1.19(16) & \mathrm{C} 34 \mathrm{~A}-\mathrm{C} 315-\mathrm{C} 316-\mathrm{C} 317 & 2.29(16) \\ \mathrm{C} 2-\mathrm{O} 1-\mathrm{C} 8 \mathrm{~A}-\mathrm{C} 8 & -178.25(10) & \mathrm{C} 34 \mathrm{~A}-\mathrm{C} 315-\mathrm{C} 316-\mathrm{N} 32 & -177.58(9) \\ \mathrm{C} 5-\mathrm{C} 4 \mathrm{~A}-\mathrm{C} 8 \mathrm{~A}-\mathrm{O} 1 & -179.57(9) & \mathrm{C} 31-\mathrm{N} 32-\mathrm{C} 316-\mathrm{C} 315 & -178.87(10) \\ \mathrm{C} 4-\mathrm{C} 4 \mathrm{~A}-\mathrm{C} 8 \mathrm{~A}-\mathrm{O} 1 & -0.41(15) & \mathrm{C} 31-\mathrm{N} 32-\mathrm{C} 316-\mathrm{C} 317 & 1.27(18) \\ \mathrm{C} 5-\mathrm{C} 4 \mathrm{~A}-\mathrm{C} 8 \mathrm{~A}-\mathrm{C} 8 & -0.15(17) & \mathrm{C} 315-\mathrm{C} 316-\mathrm{C} 317-\mathrm{C} 318 & -1.86(16) \\ \mathrm{C} 4-\mathrm{C} 4 \mathrm{~A}-\mathrm{C} 8 \mathrm{~A}-\mathrm{C} 8 & 179.00(10) & \mathrm{N} 32-\mathrm{C} 316-\mathrm{C} 317-\mathrm{C} 318 & 178.01(10) \\ \mathrm{C} 7-\mathrm{C} 8-\mathrm{C} 8 \mathrm{~A}-\mathrm{O} 1 & -179.54(10) & \mathrm{C} 316-\mathrm{C} 317-\mathrm{C} 318-\mathrm{C} 38 \mathrm{~A} & -0.20(17) \\ \mathrm{C} 7-\mathrm{C} 8-\mathrm{C} 8 \mathrm{~A}-\mathrm{C} 4 \mathrm{~A} & 1.03(18) & \mathrm{N} 311-\mathrm{C} 38 \mathrm{~A}-\mathrm{C} 318-\mathrm{C} 317 & -178.93(10) \\ \mathrm{C} 316-\mathrm{N} 32-\mathrm{C} 31-\mathrm{O} 31 & 2.43(19) & \mathrm{C} 34 \mathrm{~A}-\mathrm{C} 38 \mathrm{~A}-\mathrm{C} 318-\mathrm{C} 317 & 1.78(16) \\ \mathrm{C} 316-\mathrm{N} 32-\mathrm{C} 31-\mathrm{C} 3 & -177.72(10) & & \\ & & & \end{array}$

Hydrogen-bond geometry $\left(\AA,{ }^{\circ}\right)$

\begin{tabular}{lllll}
\hline$D-\mathrm{H} \cdots A$ & $D-\mathrm{H}$ & $\mathrm{H} \cdots A$ & $D \cdots A$ & $D-\mathrm{H} \cdots A$ \\
\hline $\mathrm{C} 314-\mathrm{H} 314 \cdots \mathrm{O} 31^{\mathrm{i}}$ & 0.95 & 2.50 & $3.278(2)$ & 139 \\
$\mathrm{C} 8-\mathrm{H} 8 \cdots \mathrm{N} 311^{\mathrm{ii}}$ & 0.95 & 2.68 & $3.394(3)$ & 133 \\
$\mathrm{C} 317-\mathrm{H} 317 \cdots \mathrm{O} 31$ & 0.95 & 2.29 & $2.903(2)$ & 122 \\
$\mathrm{~N} 32-\mathrm{H} 32 \cdots \mathrm{O} 2$ & $0.907(18)$ & $1.879(18)$ & $2.686(2)$ & $147.3(15)$ \\
\hline
\end{tabular}

Symmetry codes: (i) $x-1, y, z$; (ii) $x+1 / 2,-y+3 / 2, z-1 / 2$. 